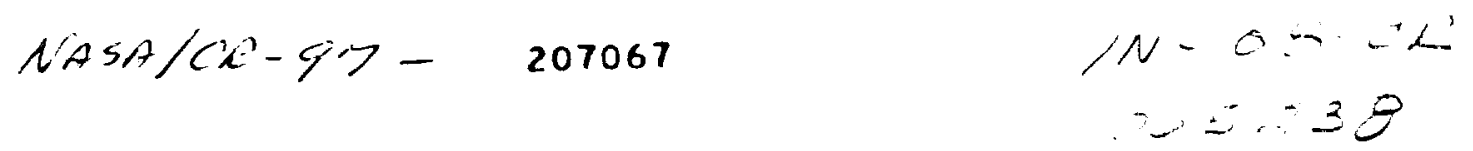

\title{
CHOOSING SENSOR CONFIGURATION FOR A FLEXIBLE STRUCTURE USING FULL CONTROL SYNTHESIS
}

\author{
Rick Lind ${ }^{1}$ \\ NASA Dryden
}

\author{
Volkan Nalbantoglu ${ }^{2}$ \\ University of Minnesota
}

\author{
Gary Balas ${ }^{3}$ \\ University of Minnesota
}

\begin{abstract}
Optimal locations and types for feedback sensors which meet design constraints and control requirements are difficult to determine. This paper introduces an approach to choosing a sensor configuration based on Full Control synthesis. A globally optimal Full Control compensator is computed for each member of a set of sensor configurations which are feasible for the plant. The sensor configuration associated with the Full Control system achieving the best closed-loop performance is chosen for feedback measurements to an output feedback controller. A flexible structure is used as an example to demonstrate this procedure. Experimental results show sensor configurations chosen to optimize the Full Control performance are effective for output feedback controllers.
\end{abstract}

\section{Introduction}

Choosing an effective set of sensor measurements is essential for designing controllers to achieve stringent performance and robustness goals. Often control requirements are not anticipated in the design stage for physical systems and sensor configurations are chosen in an ad hoc manner. Flexible structures are especially challenging systems for choosing sensor locations and types due to the large number of mode shapes. Also, tradeoffs must be met by balancing the number of sensors needed to observe the large number of closely spaced modes while simultaneously considering the added weight and cost of these additional sensors.

\footnotetext{
${ }^{1}$ NRC PostDoctoral Research Fellow, Structural Dynamics, MS 4840D/RC, Edwards, CA 93523-0273, rick.lind@dfrc.nasa.gov, Member AIAA

${ }^{2}$ Graduate Student, Aerospace Engineering and Mechanics, Minneapolis, MN 55455, nalbant Qaem.umn.edu

${ }^{3}$ Associate Professor, Aerospace Engineering and Mechanics, Minneapolis, MN 55455, balas Qaem.umn.edu, Member AIAA

${ }^{\circ}$ AIAA Guidance, Navigation and Control Conference, New Orleans LA, AIAA-97-3745, August 1997.
}

The issue of choosing sensor locations has been studied by considering grammians for observability coupled with minimizing a cost function. Skelton and DeLorenzo choose a cost function as an LQG performance metric formulated as the root mean square contribution of each sensor output. Sensors associated with small cost functions may be removed due to their low effectiveness [15].

Similar approaches are developed using modal properties. Kim and Jenkins choose a performance metric based on modal controllability weighted by the modal cost of Skelton [8]. This approach emphasizes both the degree of controllability and modal participation in the performance criteria. Lim defines a performance metric using a weighted modal projection [9]. This approach is based on a relationship between grammian singular values and modal observability. Actuator and sensor pairs are chosen with principal directions parallel to the modes with large singular values.

This paper considers an approach to choosing a sensor configuration based on Full Control synthesis. The Full Control system allows the controller to independently affect every state and error signal. Computing the optimal Full Control controller is equivalent to computing the optimal controller for a given set of sensors. Synthesis of globally optimal controllers to minimize an $\mathcal{H}_{\infty}$ or $\mu$ upper bound is formulated in the Linear Matrix Inequality (LMI) framework for Full Information feedback and extended here to the dual problem of Full Control synthesis $[12,13]$.

The issue of sensor configuration is closely associated with the issue of control design. The optimal closedloop system requires an optimal configuration of sensors and optimal gains in the compensator. Optimality in only one of these areas will restrict the achievable performance and robustness of the closed-loop system. Utilizing the Full Control system is advantageous for synthesis and analysis of sensor configuration since a Full Control compensator can be computed which is globally optimal. The procedure will not be affected by local minima associated with control synthesis. 
The technique presented in this paper considers a chosen set of sensor locations. Globally optimal Full Control compensators are computed at each of these locations to determine the maximum performance and robustness level achievable. The sensor locations chosen for implementation on the physical system correspond to the sensor locations achieving the best Full Control performance. There is no guarantee that the optimal Full Control sensor locations are equivalent to the optimal sensor locations for a general output feedback controller; however, experiments indicate this technique can choose effective configurations for a physical system.

This approach easily allows a sensor configuration to be determined by considering variations in both type and location of sensors. The plant model used to design controllers for these configurations may be generated from experimental data transfer functions or from a computational finite element model using a package such as NASTRAN. Additionally, choosing actuator configuration using globally optimal Full Information synthesis is a natural extension to this technique [10].

Sensor configurations are chosen for a flexible structure using the method described in this paper. Several sets of sensor locations are considered for feedback measurements to achieve vibration attenuation at different positions on the structure. Globally optimal Full Control compensators are computed to determine the best sensor configuration of the sets. Output feedback controllers are generated and implemented on the experimental structure using feedback from these sensor configurations.

\section{Robust Control Synthesis}

Consider a state-space description of a linear timeinvariant plant $P(s)$.

$$
\left[\begin{array}{c}
\dot{x} \\
z \\
y
\end{array}\right]=\left[\begin{array}{ccc}
A & B_{1} & B_{2} \\
C_{1} & E_{11} & E_{12} \\
C_{2} & E_{21} & E_{22}
\end{array}\right]\left[\begin{array}{l}
x \\
d \\
u
\end{array}\right]
$$

where $A \in \mathbf{R}^{n \times n}, B_{1} \in \mathbf{R}^{n \times n_{d}}, B_{2} \in \mathbf{R}^{n \times n_{w}}, C_{1} \in$ $\mathbf{R}^{n_{e} \times n}, C_{2} \in \mathbf{R}^{n_{y} \times n}$, and the $E$ matrices of appropriate similar dimensions.

Define $\mathcal{K}_{P}$ as the set of all real, rational, proper controllers, $K(s)$, which stabilize the closed-loop system. Analyzing performance using the induced $\mathcal{H}_{\infty}$ norm leads to the following minimization problem for $F_{l}(P, K)$ which is the linear fractional transformation (LFT) for the lower loop of $P$ closed with the controller $K$.

$$
\inf _{K \in \mathcal{K}} \sup _{\omega \in \mathbb{R}} \bar{\sigma}\left[F_{l}(P(j \omega), K(\gamma \omega))\right]=\inf _{K \in \mathcal{K}}\left\|F_{l}(P, K)\right\|_{\infty}
$$

This is an $\mathcal{H}_{\infty}$ optimal controller synthesis problem which has been solved using state-space equations $[3,5]$.
The structured singular value, $\mu$, can be used to determine robustness of the closed-loop system to structured modeling uncertainty and the achievable performance level in the presence of real and complex uncertainty. The uncertainty description is structured with two types of blocks. The blocks are repeated scalar or full block matrices. Let integers $m, n, p$ define the number of real scalar, complex scalar, and complex full blocks. Define integers $R_{1}, \ldots, R_{m}$ such that the $i^{\text {th }}$ repeated scalar block of real parametric uncertainty is of dimension $R_{i} \times R_{i}$. Define similar integers $C_{1}, \ldots, C_{n}$ to denote the dimension of the complex repeated scalar blocks. The structured uncertainty description $\Delta$ is assumed to be norm bounded and belonging to the following set.

$$
\begin{gathered}
\Delta=\left\{\operatorname{diag}\left(\delta_{1}^{R} I_{R_{1}} \ldots \delta_{m}^{R} I_{R_{m}} \delta_{1}^{C} I_{C_{1}} \ldots \delta_{n}^{C} I_{C_{n}} \Delta_{1} \ldots \Delta_{p}\right)\right. \\
\left.: \delta_{i}^{R} \in \mathbf{R}, \delta_{i}^{C} \in \mathbf{C}, \Delta_{i} \in \mathbf{C}^{c_{i} \times c_{i}}\right\}
\end{gathered}
$$

Real parametric uncertainty is allowed to enter the problem as scalar or repeated scalar blocks. Complex uncertainty enters the problem as scalar, repeated scalar or full blocks.

The function $\mu$ is defined as

$$
\mu_{\Delta}(P)=\frac{1}{\min _{\Delta}\{\tilde{\sigma}(\Delta): \operatorname{det}(I-P \Delta)=0\}}
$$

with $\mu(P)=0$ if no $\Delta$ exists such that $\operatorname{det}(I-P \Delta)=0$.

Upper and lower bounds for $\mu$ have bee derived which utilize two sets of scaling matrices which are structured similar to the uncertainty block structures.

$$
\begin{gathered}
\mathcal{D}=\left\{\operatorname{diag}\left(D_{1}^{R}, \ldots, D_{m}^{R}, D_{1}^{C}, \ldots, D_{n}^{C}, d_{1}^{c} I_{c_{1}}, \ldots, d_{p}^{c} I_{c_{p}}\right)\right. \\
\left.: 0<D=D^{*}, D_{i}^{R} \in \mathbf{C}^{R_{i} \times R_{i}}, D_{i}^{C} \in \mathbf{C}^{C_{i} \times C_{i}}, d_{i}^{c} \in \mathbf{C}\right\}
\end{gathered}
$$

The second set of scalings in $\mathcal{G}$ affect only the real parametric uncertainty blocks.

$$
\mathcal{G}=\left\{\operatorname{diag}\left(G_{1}, \ldots, G_{m}, 0, \ldots, 0\right): G_{i} \in \mathbf{C}^{R_{i} \times R_{i}}\right\}
$$

An upper bound for $\mu$ is computed as an optimization [4]. The real/complex $\mu$ upper bound reduces to the well known complex $\mu$ upper bound when there are no real parametric uncertainty blocks.

$$
\mu(P) \leq \inf _{\substack{G \in \mathcal{G} \\ D \in \mathcal{D}}} \bar{\sigma}\left(\left(D P D^{-1}+\jmath G\right)\left(I+G^{2}\right)^{\frac{1}{2}}\right)
$$

The structured singular value provides a measure of robustness in the presence of the defined structured uncertainty. The $\mathcal{D}$ and $\mathcal{G}$ are restricted to be constant matrices to scale with time-varying uncertainty in this paper. The objective of control design is to maximize robust performance which corresponds to minimizing $\mu$ in this framework. 


\section{Feasibility : Output Feedback System}

$\mathcal{H}_{\infty}$ control synthesis involves iterating over a set of feasibility conditions. These conditions determine whether a controller exists that achieves a desired closed-loop $\mathcal{H}_{\infty}$ norm value. A standard bisection search can be used to find the lowest achievable norm value to within a given accuracy. The optimal controller is computed using elements of the plant and the solutions to the feasibility conditions.

The controller feasibility and synthesis may be formulated as state-space equations or in the LMI framework $[6,7]$. The feasibility conditions in the state-space framework are two Riccati equations. The comparable feasibility conditions in the LMI framework are generated by applying the Bounded Real Lemma and considering orthogonal subspaces to matrix elements.

A separate LMI formulation is developed for computing optimal full information controllers $[12,13]$. This approach uses algebraic arguments to demonstrate a constant matrix condition which is equivalent to the statespace $\mathcal{H}_{\infty}$ control problem. LMI feasibility conditions are generated using a variant of Parrott's theorem [14].

This paper will adopt a standard for denoting plant matrices for ease of notation and convenience in theorems. Denote $P$ as the continuous-time state-space plant matrix with the elements $P(s)=\{A, B, C, D\}$. Denote $M_{P}$ as the constant matrix whose entries are comprised of the state-space elements of $P$.

$$
P=D+C(s I-A)^{-1} B \Leftrightarrow M_{P}=\left[\begin{array}{cc}
A & B \\
C & D
\end{array}\right]
$$

The LMI feasibility conditions utilize a matrix $T_{\alpha}$ which is formulated for a real scalar $\alpha>0$.

$$
T_{\alpha}=\left[\begin{array}{cc}
I & \sqrt{2 \alpha} I \\
\sqrt{2 \alpha} I & \alpha I
\end{array}\right]
$$

This matrix is used to compute the following star product LFT with $\hat{A}=(I+\alpha A)^{-1}$ defined for notational convenience.

$$
F_{s}\left(T_{\alpha}, M_{P}\right)=\left[\begin{array}{cc}
(I+\alpha A) \hat{A} & \sqrt{2 \alpha} \hat{A} B \\
\sqrt{2 \alpha} C \hat{A} & E+\alpha C \hat{A} B
\end{array}\right]
$$

Computing the star product with $T_{\alpha}$ has several important properties. The most immediately noticed property is the relationship between the star product and the bilinear transformation. The matrix $\hat{P}=F_{s}\left(T_{\alpha}, P\right)$ is the discrete-time formulation of the continuous-time plant $P$. The star product also has a commutation property such that $F_{s}\left(T_{\alpha}, F_{l}(P, K)\right)=F_{l}\left(P, F_{s}\left(T_{\alpha}, K\right)\right)$.

The following theorem demonstrates a constant matrix condition, formulated using the star product, which is equivalent to an $\mathcal{H}_{\infty}$ condition [13].
Theorem 1 Given the state-space plant $P(s)$ and associated constant matrix $M_{P}$ along with the set $\mathcal{D}$ of scaling matrices, then the following are equivalent.

1. There exists $D \in \mathcal{D}$ and stabilizing $K \in \mathcal{K}_{P}$ such that

$$
\left\|D^{\frac{1}{2}} F_{l}(P, K) D^{-\frac{1}{2}}\right\|_{\infty}<1
$$

2. There exists $D \in \mathcal{D}$ and stabilizing $K \in \mathcal{K}_{P}$ along with real $X=X^{T}>0$ such that with $Z=\operatorname{diag}(X, D)$,

$$
\bar{\sigma}\left(Z^{\frac{1}{2}} F_{s}\left(T_{\alpha}, F_{l}\left(M_{P}, K\right)\right) Z^{-\frac{1}{2}}\right)<1
$$

3. There exists $D \in \mathcal{D}$ and stabilizing $K \in \mathcal{K}_{P}$ along with real $X=X^{T}>0$ such that with $Z=\operatorname{diag}(X, D)$,

$$
\bar{\sigma}\left(Z^{\frac{1}{2}} F_{l}\left(F_{a}\left(T_{\alpha}, M_{P}\right), K\right) Z^{-\frac{1}{2}}\right)<1
$$

Now perform a change of variables. Denote $\{R, U, V, T\}$ as elements of the constant matrix term involving the star product $F_{s}\left(T_{\alpha}, M_{P}\right)$. Introduce $Q$ to replace $K(I+$ $T K)^{-1}$ in the closed-loop LFT for notational convenience.

$F_{l}\left(F_{s}\left(T_{\alpha}, M_{P}\right), K\right)=F_{l}\left(\left[\begin{array}{ll}R & U \\ V & T\end{array}\right], K\right)=R+U Q V$

The final theorem presents the pair of LMI optimizations that represent the $\mathcal{H}_{\infty}$ controller feasibility condition for a general output feedback system. The variant of Parrott's theorem is applied to the constant matrix condition involving the maximum singular value.

Theorem 2 Given the state-space plant $P(s)$ and associated constant matrix $M_{P}$ with the star product elements $F_{l}\left(F_{s}\left(T_{\alpha}, M_{P}\right), K\right)=R+U Q V$ along with the set $\mathcal{D}$ of scaling matrices, then the following are equivalent.

1. There exists stabilizing $K \in \mathcal{K}_{P}$ and $D \in \mathcal{D}$ such that

$$
\left\|D^{\frac{1}{2}} F_{l}(P, K) D^{-\frac{1}{2}}\right\|_{\infty}<1
$$

2. There exists stabilizing $K \in \mathcal{K}_{P}$ and $D \in \mathcal{D}$ along with real $X=X^{T}>0$ such that with $Z=\operatorname{diag}(X, D)$,

$$
\bar{\sigma}\left(Z^{\frac{1}{2}}(R+U Q V) Z^{-\frac{1}{2}}\right)<1
$$

3. There exists stabilizing $K \in \mathcal{K}_{P}$ and $D \in \mathcal{D}$ along with real $X=X^{T}>0$ such that with $Z=\operatorname{diag}(X, D)$,

$$
\begin{gathered}
\bar{\lambda}\left(U_{\perp}^{T}\left(R Z^{-1} R^{T}-Z^{-1}\right) U_{\perp}\right)<0 \\
\bar{\lambda}\left(V_{\perp}\left(R^{T} Z R-Z\right) V_{\perp}^{T}\right)<0
\end{gathered}
$$




\section{Feasibility : Optimal Full Control}

Consider the state-space Full Control plant $P_{f c}$.

$$
P_{f c}=\left[\begin{array}{c|ccc}
A & B & I & 0 \\
\hline C_{1} & D_{11} & 0 & I \\
C_{2} & D_{21} & 0 & 0
\end{array}\right]
$$

Define $M_{P_{f c}}$ as the constant matrix associated with the state-space elements of $P_{f c}$. Formulate the $R, U, V$ elements of the star product term $F_{s}\left(T_{\alpha}, M_{P_{g_{c}}}\right)$ using the term $\hat{A}=(I+\alpha A)^{-1}$ for notational convenience.

$$
\begin{aligned}
& R=\left[\begin{array}{cc}
(I+\alpha A) \hat{A} & \sqrt{2 \alpha} \hat{A} B_{1} \\
\sqrt{2 \alpha} C_{1} \hat{A} & E_{11}+\alpha C_{1} \hat{A} B_{1}
\end{array}\right] \\
& U=\left[\begin{array}{cc}
\sqrt{2 \alpha} \hat{A} B_{1} & 0 \\
\alpha C_{1} \hat{A} & I
\end{array}\right] \\
& V=\left[\begin{array}{cc}
\sqrt{2 \alpha} C_{2} \hat{A} & E_{12}+\alpha C_{2} \hat{A} B_{1}
\end{array}\right]
\end{aligned}
$$

The matrix $U$ is square and invertible for the Full Control system. This full rank condition is anticipated by the complete controllability of this system. A linearly independent set of control vectors are available to affect the states and error outputs of the plant. Correspondingly, the perpendicular subspace, $U_{\perp}$, utilized in the LMI conditions for $\mathcal{H}_{\infty}$ controller feasibility is null.

The feasibility condition for existence of an $\mathcal{H}_{\infty}$ controller for Full Control feedback is reduced to a single LMI. The LMI involving $U_{\perp}$ in Theorem 2 is vacuous and automatically satisfied. The remaining LMI involving variables $V$ and $V_{\perp}$ constitutes the only condition for Full Control feasibility as demonstrated in Theorem 3.

Theorem 3 Given the Full Control plant $P_{f c}$ and scaling set $\mathcal{D}$, define the following :

1. The augmented scaling matrices $\mathcal{Z}$

$$
\mathcal{Z}=\left\{\left[\begin{array}{cc}
X & 0 \\
0 & D
\end{array}\right]: 0<X=X^{T} \in \mathbf{R}^{n \times n}, D \in \mathcal{D}\right\}
$$

2. Real scalar $\alpha>0$, so that $(I-\alpha A)$ is invertible

3. $R$ and $V$ as defined above

4. $V_{\perp}$ such that $V^{T} V_{\perp}=0$ and $\left[\begin{array}{c}V \\ V_{\perp}\end{array}\right]$ is invertible

Then, there exists a stabilizing $K \in \mathcal{K}_{P_{f_{\mathrm{c}}}}$ and a constant $D \in \mathcal{D}$ such that

$$
\left\|D^{\frac{1}{2}} F_{l}\left(P_{f c}, K\right) D^{-\frac{1}{2}}\right\|_{\infty}<1
$$

if and only if the following convex set is nonempty.

$$
\left\{Z \in \mathcal{Z}: \lambda_{\max }\left[V_{\perp}\left(R^{*} Z R-Z\right) V_{\perp}^{*}\right]<0\right\} \neq\{\emptyset\}
$$

This formulation is easily extended to account for real parametric uncertainty [2]. The maximum singular value condition in Theorem 2 is replaced with the corresponding condition from the real/complex $\mu$ upper bound. Consider this condition for the matrix system $R+U Q V$ including the additional scaling matrices $\mathcal{G}$.

$$
\begin{aligned}
\mu(R+U Q V) & \\
\leq & \bar{\sigma}\left(\left(D(R+U Q V) D^{-1}+\jmath G\right)\left(I+G^{2}\right)^{-\frac{1}{2}}\right) \\
\leq & \bar{\sigma}(\bar{R}+\bar{U} Q \bar{V}) \\
\text { where } \quad \bar{R} & =\left(D R D^{-1}+\jmath G\right)\left(I+G^{2}\right)^{-\frac{1}{2}} \\
\bar{U} & =D U \\
\bar{V} & =V D^{-1}\left(I+G^{2}\right)^{-\frac{1}{2}}
\end{aligned}
$$

The variant of Parrott's theorem can be applied to this new singular value condition in the variables $\bar{R}, \bar{U}$ and $\bar{V}$. The new matrix $\bar{U}$ retains the desired full rank condition since both $D$ and $U$ are invertible. Thus, a single LMI represents the feasibility condition. Consider this LMI.

$$
\begin{gathered}
\bar{\lambda}\left(\bar{V}_{\perp}\left(\bar{R}^{*} \bar{R}-I\right) V_{\perp}^{*}\right) \\
=\bar{\lambda}\left(V _ { \perp } D \left(\left(D^{-1} R^{*} D-\jmath G\right)\left(D R D^{-1}+\jmath G\right)\right.\right. \\
\left.\left.\quad-\left(I+G^{2}\right)\right) D V_{\perp}^{*}\right) \\
=\bar{\lambda}\left(V_{\perp}\left(R^{*} \bar{D} R+\jmath\left(R^{*} \bar{G}-\bar{G} R\right)-\bar{D}\right) V_{\perp}^{*}\right) \\
\text { where } \quad \bar{D}=D^{2} \in \mathcal{D} \\
\bar{G}=D G D \in \mathcal{G}
\end{gathered}
$$

This feasibility condition to determine existence of a controller that satisfies a closed-loop $\mu$ condition may be less conservative than the previous condition since it directly accounts for real parametric uncertainty. Theorem 4 combines Theorem 3 with the real/complex $\mu$ bound.

Theorem 4 Given the Full Control plant $P_{f c}$ and scaling sets $\mathcal{D}, \mathcal{G}$ define the following :

1. The augmented scaling matrices $\mathcal{Z}_{D}$

$$
\mathcal{Z}_{\mathcal{D}}=\left\{\left[\begin{array}{cc}
X & 0 \\
0 & D
\end{array}\right]: 0<X=X^{T} \in \mathbf{R}^{n \times n}, D \in \mathcal{D}\right\}
$$

2. The augmented scaling matrices $\mathcal{Z}_{G}$

$$
\mathcal{Z}_{\mathcal{G}}=\left\{\left[\begin{array}{cc}
0 & 0 \\
0 & G
\end{array}\right]: G \in \mathcal{G}\right\}
$$

3. $R, V, V_{\perp}$ and $\alpha$ as defined in Theorem 3.

Then, there exists a stabilizing $K \in \mathcal{K}_{P_{f_{\mathrm{c}}}}$ such that

$$
\mu\left(F_{l}\left(P_{f c}, K\right)\right)<1
$$

if the following convex set is nonempty.

$$
\begin{aligned}
& \left\{Z_{D} \in \mathcal{Z}_{\mathcal{D}}, Z_{G} \in \mathcal{Z}_{\mathcal{G}}:\right. \\
& \left.\quad \bar{\lambda}\left[V_{\perp}\left(R^{*} Z_{D} R+\jmath\left(R^{*} Z_{G}-Z_{G} R\right)-Z_{D}\right) V_{\perp}^{*}\right]<0\right\}
\end{aligned}
$$




\section{Choosing Sensor Configuration}

Synthesis of optimal Full Control compensators can be used to determine efficient sensor configurations. The Full Control system allows the controller to independently affect every state and error signal. The previous section demonstrates globally optimal controllers can be computed for the Full Control system. Computing optimal Full Control compensators is equivalent to computing the optimal controller for a given set of sensors.

An optimal sensor configuration can be chosen by minimizing the achievable Full Control performance level with respect to a set of possible sensor configurations. Plant models are generated for each sensor configuration under consideration and a Full Control compensator is computed for each plant using Theorem 4. The optimal sensor configuration chosen with this method may not be globally optimal over every possible sensor location in the system; rather, it is optimal with respect to the considered set of locations.

The following algorithm demonstrates this procedure.

\section{Algorithm 1}

- Define state-space elements of transfer function from disturbances to errors.

$$
P=\left[\begin{array}{cc}
A & B_{1} \\
C_{1} & D_{11}
\end{array}\right]
$$

- Define set of $n$ sensor configurations.

$$
\left[\begin{array}{cc}
C_{2}^{1} & D_{21}^{1} \\
\vdots & \\
C_{2}^{n} & D_{21}^{n}
\end{array}\right]
$$

for $i=1: n\{$

$$
\begin{aligned}
& \text { Formulate } P_{f c}^{i}=\left[\begin{array}{cccc}
A & B_{1} & I & 0 \\
C_{1} & D_{11} & 0 & I \\
C_{2}^{i} & D_{21}^{i} & 0 & 0
\end{array}\right] \\
& \text { Compute } \gamma_{i}=\inf _{K \in \mathcal{K}} \mu\left(F_{l}\left(P_{f c}^{i}, K\right)\right)
\end{aligned}
$$

)

Choose sensor configumation $\left[\begin{array}{ll}C_{2}^{j} & D_{21}^{j}\end{array}\right]$ corresponding to $\gamma_{j}=\min _{i} \gamma_{i}$.
The plant model and associated set of sensor configurations used in this algorithm may be generated using computational packages, such as NASTRAN, or experimentally derived transfer functions. Finite element models give the freedom to easily compute a large number of sensor configurations but may be subject to modeling errors. Experimental data gives a more accurate representation of the true system but it may be difficult to physically reposition a large number of sensors and identify models for each location.

Location, type, and number of sensors used in each configuration, along with the total number of configurations to be considered in the set, may be chosen using several criteria. The set may reflect physical requirements limiting the sensors to select configurations due to size of the sensor, direction of sensing, wiring connections and weight restrictions. The set may also be chosen based on a priori knowledge of the system and properties such as symmetries, mode shapes, and previous control design experience.

This algorithm is not guaranteed to generate a globally optimal sensor configuration for output feedback controllers. The computed optimal sensor location may not even be optimal among the discrete set of sensor configurations when considering an output feedback controller. The computed sensor configuration is only optimal with respect to a Full Control compensator; however, there are many plants which can effectively utilize this method. If the dominant modes affecting the achievable performance are controllable, or nearly so, then the physical actuator sets used for output feedback controllers will be able to achieve, or nearly so, the performance of the Full Control compensator.

A similar procedure can be formulated based on minimizing the achievable performance level of output feedback controllers; however, there are advantages to using Full Control synthesis. The Full Control compensators are guaranteed to be globally optimal while output feedback controllers, computed with methods such as $D-K$ iteration, are only locally optimal. A poor local minimum may capture the output feedback controller synthesis for a given sensor configuration. The resulting optimal sensor configuration would be incorrectly computed due to deficiencies in the control synthesis procedure.

Additionally, the Full Control synthesis is an LMI which is easily solved with no user interaction using convex optimization algorithms. $D-K$ iteration requires the user to monitor the process and select weighting functions. Successive $D-K$ iterations might produce better, or worse, performing controllers depending on these weight selections and initial conditions. 


\section{Flexible Structure : Model}

Sensor configurations are chosen for vibration attenuation of an experimental flexible structure. The flexible structure is constructed at the Dynamics and Controls Labomatory in the Department of Aerospace Engineering and Mechanics at the University of Minnesota. This structure models a space truss for potential satellite and space platform applications. The structure is designed to place 12 lightly damped modes between 0 and $100 \mathrm{rad} / \mathrm{sec}$. The flexible structure is represented in Figure 1 and Figure 2.

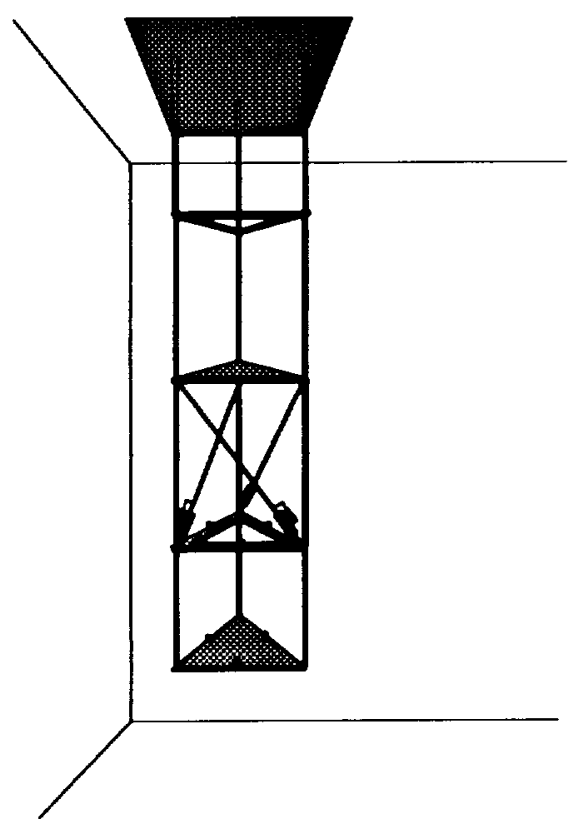

Figure 1: University of Minnesota Flexible Structure

The structure consists of a rigidly held fixed top plate and four hanging bays numbered 1 through 4 with Bay 1 being at the top of the structure and Bay 4 at the bottom. Each bay contains an aluminum plate and thin hollow rods connecting corners of neighboring plates. The top four plates are spaced $.62 \mathrm{~m}$ apart while the bottom plate is $.47 \mathrm{~m}$ below the third bay. Plates in Bay 2 and Bay 4 are triangular frames while the others are solid.

The actuators for control are contained in Bay 3. Three actuators are colocated along diagonal rods connecting the top and bottom plates of this bay. These force actuators are voice-coil type actuators produced by Northern Magnetics as ML3-1310-020LB with a limit of \pm 2 pounds of force. The working linear stroke is $\pm \frac{1}{2}$ inch with an effective bandwidth of $200 \mathrm{~Hz}$.

Linear displacement sensors are colocated along the direction of the force actuators in Bay 3. These sensors are Trans-Tek 0242 type sensors with a working range of $\pm \frac{1}{2}$ inch. They have zero hysteresis and are linear to within $\pm .5 \%$ up to the bandwidth of $100 \mathrm{~Hz}$.
Accelerometers may be placed along the edges of any plate in any Bay. These accelerometers are ICSensor 3145-002 with a bandwidth of $300 \mathrm{~Hz}$. These sensors are aligned along the horizontal component of an actuator and do not measure any vertical component of movement.

Figure 2 shows the control elements in Bay 3. The colocated actuators and displacement sensors are seen along the diagonal rods. The accelerometers are placed atop the horizontal edges of the triangular frame plate.

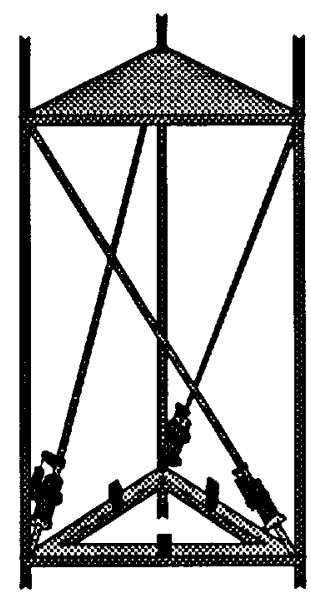

Figure 2: Control Elements in Bay 3

An analytical model is generated for the structure. A NASTRAN model is available; however, this paper uses experimental data to formulate the model. Experimental transfer functions are computed from the actuators to the accelerometers on Bays 3 and 4 by commanding sinusoids of varying frequency to the actuators. System identification algorithms based on curve fitting techniques and model reduction via balanced realization computes a $38^{\text {th }}$ order model. Transfer functions plots of the openloop peak gain for Bay 3 and Bay 4 accelerometers is given in Figure 3.

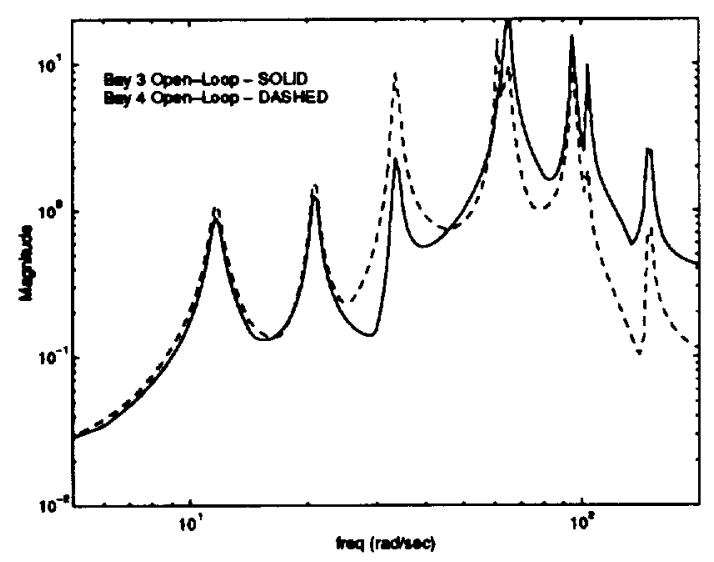

Figure 3: Open-Loop Peak Gains from Bay 3 Actuators to Bay 3 and Bay 4 Accelerometers 
It is desired to formulate controllers to attenuate vibration and lower the peak gains of the open-loop system. Accelerometers are used as the performance signals with a peak gain of approximately 10 for the $65 \mathrm{rad} / \mathrm{sec}$ mode. Bay 3 accelerometers are located in the same bay as the actuators so it is anticipated more performance can be achieved for these sensors. Bay 3 attenuation is desired to be approximately 4.6 while the performance request for Bay 4 is a factor of 3.5 for attenuation. Performance weightings, $W_{\text {perf }}^{\text {bay3 }}$ and $W_{\text {perf }}^{\text {bav4 }}$, are included with the accelerometer error signals to specify the desired attenuation levels.

$$
W_{\text {perf }}^{\text {bay3 }}=\frac{4.6}{10} \quad W_{\text {perf }}^{\text {bay4 }}=\frac{3.5}{10}
$$

Additive uncertainty between the control inputs and sensor measurements is included to account for unmodeled dynamics and neglected high frequency modes. A dynamic weighting, $W_{a d d}$, is affected on each displacement and accelerometer sensor for feedback.

$$
W_{a d d}=4 \frac{s^{2}-72 s+4790}{s^{2}-205 s+28910}
$$

The physical actuator positions are affected by a disturbance input. A constant weighting, $W_{\text {dist }}=.5$, is included to normalize the disturbance signal affecting each control channel. Sensor noise is also included in the system to affect the accelerometer feedback measurements. Constant weightings of $W_{\text {noise }}=.01$ are included to normalize the noise affecting each sensor.

The magnitude of the control signal for each physical actuator is included as a performance error to limit the amount of control actuation. A weighting of $W_{a c t}=.2$ is used as the performance penalty for each actuator.

The open-loop flexible structure model with uncertainty blocks and weightings in given in Figure 4.

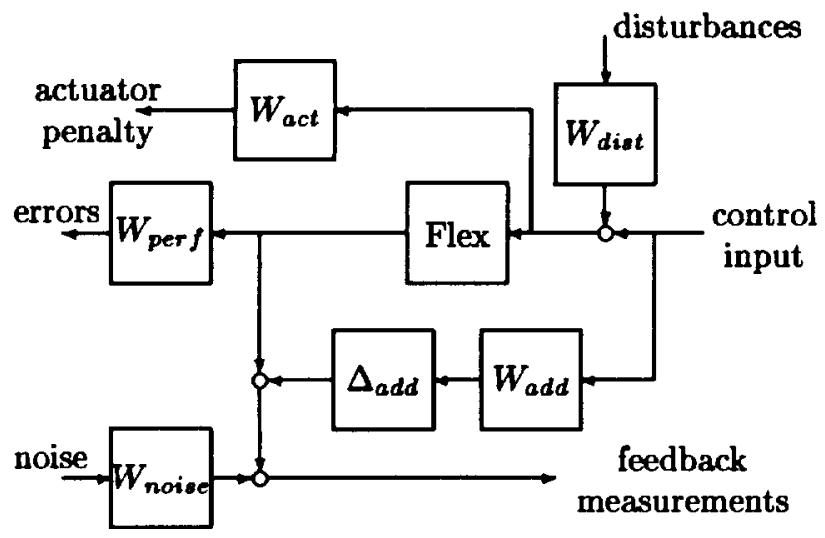

Figure 4: Flexible Structure Block Diagram
Full Control compensators are computed for systems derived as subsets of the flexible structure model in Figure 4 . This block diagram is designed with 6 accelerometers and 3 displacement sensors for performance errors and feedback measurements. These 9 sensors are divided into 3 groups of 3 with the following notation.

$$
\begin{aligned}
& \mathbf{3}_{1}^{d}, \mathbf{3}_{2}^{d}, \mathbf{3}_{3}^{d}-\text { displacement sensors in Bay } 3 \\
& \mathbf{3}_{1}, \mathbf{3}_{2}, \mathbf{3}_{3}-\text { acceleration sensors in Bay } 3 \\
& \mathbf{4}_{1}, \mathbf{4}_{2}, \mathbf{4}_{3}-\text { acceleration sensors in Bay } 4
\end{aligned}
$$

The subscript on the sensor designations indicates the horizontal component of the sensing direction. The accelerometers are placed along edges of the horizontal plates while the displacement sensors are colocated along the diagonal rods connecting corners of neighboring plates with the same horizontal direction of sensing as the accelerometers. Sensors $3_{1}^{d}, 3_{1}$ and $4_{1}$ are located along the same edges of plates on the same side of the structure and consequently have the same horizontal direction of sensing.

The uncertainty structure for the system in Figure 4 has a single uncertainty block. This block represents additive uncertainty on the sensor signals used for feedback measurements to the controller. This uncertainty, $\Delta_{\text {add }}$, is a complex operator to allow variations in both magnitude and phase. $\Delta_{a d d}$ is treated as an unstructured full block uncertainty for controller synthesis.

A performance block will also be included in the controller synthesis procedure. This block relates the noise and disturbance inputs to the performance errors. There are 3 noise disturbances affecting the 3 physical actuators and 9 noise inputs for the sensors. The performance errors are composed of 3 penalties on the amount of actuation and 6 weightings on the accelerometers.

Initial controllers are synthesized to determine the type of sensors to use for feedback. Full Control compensators are computed for the system with Bay 3 accelerometers, $\mathbf{3}_{1}, \mathbf{3}_{2}, \mathbf{3}_{3}$, used as the error signals to be minimized and feedback measurements of either the Bay 3 displacements, $3_{1}^{d}, 3_{2}^{d}, 3_{3}^{d}$, or the Bay 3 accelerations, $3_{1}, 3_{2}, 3_{3}$. The optimal achievable $\mathcal{H}_{\infty}$ norm was lower using the accelerometers than the displacement sensors for feedback. This result agrees with previous analysis of the structure indicating the accelerometers are generally more effective than the displacement sensors for vibration attenuating controllers [11]. The control designs presented in this paper will ignore the displacement sensors and only utilize the accelerometers. 
Full Control compensators are computed for several configurations of accelerometers. The performance errors used for vibration attenuation are chosen to be either the Bay 3 accelerometers, $\left\{3_{1}, 3_{2}, 3_{3}\right\}$, or the Bay 4 accelerometers, $\left\{\mathbf{4}_{1}, \mathbf{4}_{2}, \mathbf{4}_{3}\right\}$. Several combinations of these sensors are used for feedback measurements with either 2 or 3 sensors in each combination. The number of combinations is reduced by symmetry arguments which indicate $\left\{3_{1}, 3_{2}\right\}$ should be as effective as $\left\{3_{1}, 3_{3}\right\}$ and $\left\{3_{2}, 3_{3}\right\}$. The achievable $\mu$ performance levels for the optimal Full Control compensators are given in Table 1.

\begin{tabular}{|c|c|c|}
\hline $\begin{array}{c}\text { Feedback } \\
\text { Accelerometers }\end{array}$ & $\begin{array}{c}\text { Bay 3 } \\
\text { Performance } \\
\left\|\mathbf{3}_{1}, \mathbf{3}_{2}, \mathbf{3}_{3}\right\|_{\infty}\end{array}$ & $\begin{array}{c}\text { Bay } 4 \\
\text { Performance } \\
\left\|4_{1}, 4_{2}, 4_{3}\right\|_{\infty}\end{array}$ \\
\hline $\mathbf{3}_{\mathbf{1}}, \mathbf{3}_{\mathbf{2}}$ & 1.378 & 3.031 \\
\hline $3_{1}, 3_{2}, 3_{3}$ & 0.299 & 0.719 \\
\hline $4_{1}, 4_{2}$ & 1.641 & 0.656 \\
\hline $4_{1}, 4_{2}, 4_{3}$ & 0.299 & 0.511 \\
\hline $3_{1}, 4_{1}$ & 3.281 & 1.312 \\
\hline $3_{1}, 4_{2}$ & 1.641 & 0.609 \\
\hline
\end{tabular}

Table 1: Achievable Full Control $\mu$ Performance Levels

\section{Flexible Structure : Bay 3 Attenuation}

Analysis of the first performance column of Table 1 indicates the effectiveness of various sensor configurations for providing feedback to control Bay 3 accelerometers.

The most effective sensor configuration for Bay 3 vibration attenuation is to use three sensors in the same bay. Feedback configurations using all Bay 3 sensors or using all Bay 4 sensors achieve $\mu=.299$ for Full Control closed-loop performance. These performance levels are similar since the set of sensors in each bay is able to observe the dynamics of Bay 3. Each set of accelerometers is able to provide sufficient information to the controller to attenuate the Bay 3 vibration responses.

Restricting the feedback to only two sensors significantly decreases the optimal performance level. The $\mu$ values increase by approximately a factor of 4 when using $\left\{3_{1}, 3_{2}\right\}$ as compared to $\left\{3_{1}, 3_{2}, 3_{3}\right\}$ for feedback. The performance decreases even more if the two sensor are aligned in the same direction. The $\mu$ of 3.28 is for $\left\{3_{1}, 4_{1}\right\}$ is twice the $\mu=1.64$ value achieved when using $\left\{3_{1}, 4_{2}\right\}$.

Output feedback controllers are generated using $D-K$ iteration for vibration attenuation of the Bay 3 accelerometers. Separate controllers are designed for 2 different feedback configurations. The first controller, $K_{3}^{3}$, will use the 3 sensors in Bay 3 to control vibration in Bay 3. The second controller, $K_{4}^{3}$ will feedback the 3 sensors in Bay 4 to attenuate vibration in Bay 3.
Robust stability is plotted for each controller in Figure 5. The peak robust stability $\mu$ values are .445 for $K_{3}^{3}$ and .456 for $K_{4}^{3} . \mu$ is less than one for each controller to indicate the desired robustness objectives are achieved.

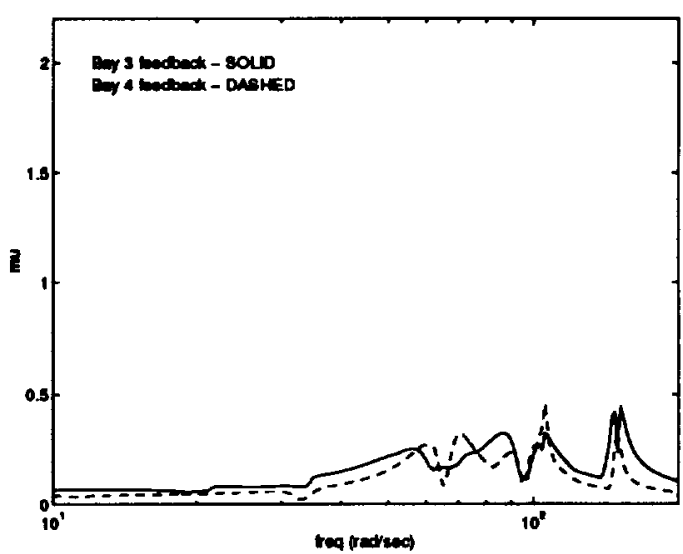

Figure 5: $\boldsymbol{\mu}$ for Robust Stability

Nominal performance is also calculated for each controller as 1.152 for $K_{3}^{3}$ and 1.880 for $K_{4}^{3}$. The weighted norms greater than 1 indicate neither controller is able to achieve the desired performance objectives. $\mu$ for nominal performance is plotted in Figure 6.

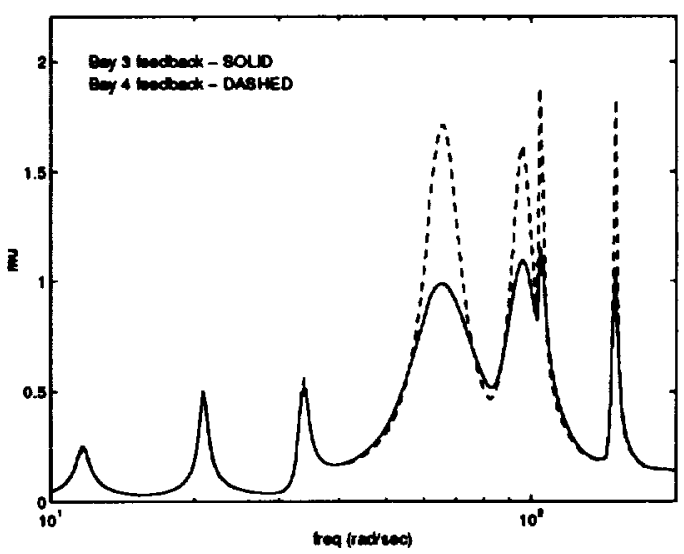

Figure 6: $\mu$ for Nominal Performance

The robust performance $\mu$ values for each controller is given in Figure 7. The $\mu$ upper bounds are computed as 1.296 for $K_{3}^{3}$ and 2.065 for $K_{4}^{3}$. Each controller gives a peak $\mu$ greater than 1 indicating robust performance is not achieved for either output feedback controller.

The robust performance $\mu$ plots are of similar shape for each controller with peaks at $104 \mathrm{rad} / \mathrm{sec}$ even though $\mu$ for $K_{4}^{3}$ is much higher. Both controllers are driven by meeting the performance goals as evidenced by Figure 6 . The open-loop gains in Figure 3 are smaller from the actuators to the Bay 4 sensors as compared to the Bay 3 sensors. $K_{4}^{3}$ is unable to increase the controller gains to match the performance of $K_{3}^{3}$ due to the penalty on the amount of control actuation. 


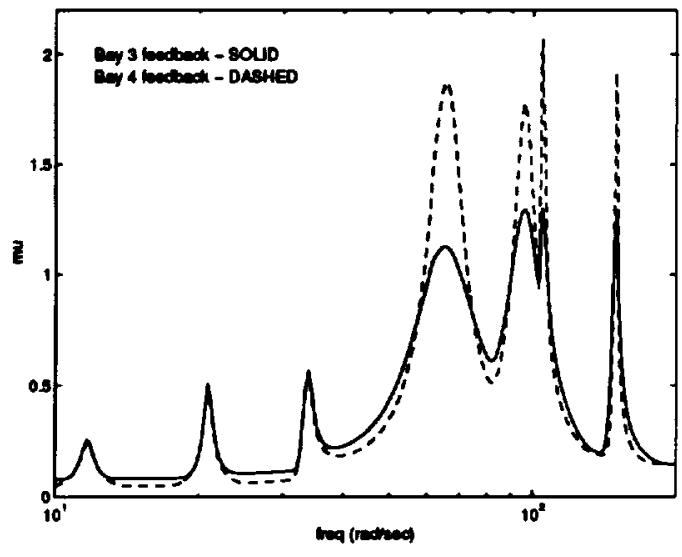

Figure 7: $\mu$ for Robust Performance

The Full Control synthesis results indicated controllers could be computed which achieve similar performance levels using either Bay 3 or Bay 4 feedback. There are several possible explanations to account for the poor performance of $K_{4}^{3}$ in comparison to $K_{3}^{3}$. The Full Control results are based on a globally optimal controller while $D-K$ iteration may have computed a $K_{4}^{3}$ far from optimal. Also, the optimal Full Control compensator may be realized as a constant gain controller while $K_{4}^{3}$ was greatly affected by bandwidth constraints.

Peak gains of the experimental closed-loop transfer functions are plotted in Figure 8. $K_{3}^{3}$ provides better attenuation for the modal response at $64 \mathrm{rad} / \mathrm{sec}$ which agrees with Figure 7. Neither controller is able to provide the desired attenuation above $104 \mathrm{rad} / \mathrm{sec}$.

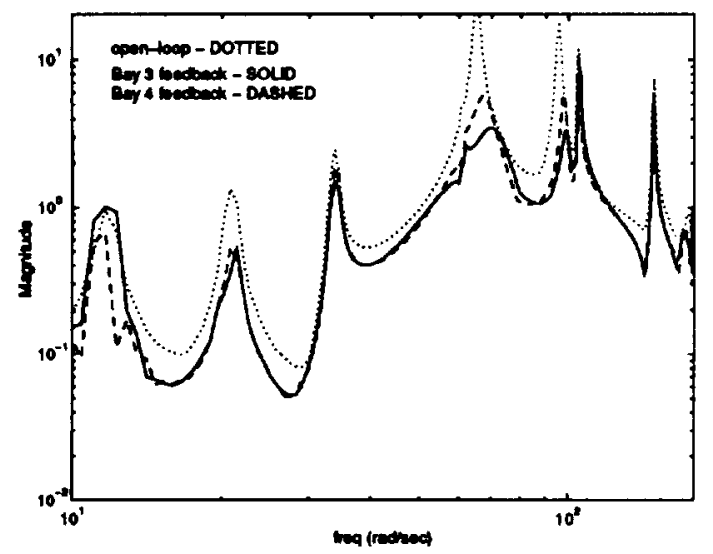

Figure 8: Experimental Closed-Loop Peak Gains for Bay 3 Accelerometers with $K_{3}^{3}(-)$ and $K_{4}^{3}(--)$

The similar performance levels on the physical structure somewhat contradict the output feedback $\mu$ analysis, which anticipated $K_{3}^{3}$ should provide $40 \%$ better attenuation, but agree more closely with the Full Control analysis, which anticipated each controller should provide similar attenuation levels.
Flexible Structure : Bay 4 Attenuation

The achievable performance levels of optimal Full Control compensators to attenuate vibrations measured by Bay 4 accelerometers for various sensor configurations is given in the last column of Table 1.

These performance levels clearly indicate some measure of the Bay 4 accelerometers is required for adequate attenuation of Bay 4 vibrations. Synthesizing a Full Control compensator using the entire set of Bay 3 accelerometers achieves a $\mu$ value of .719 while a $\mu$ value is .511 is achieved using the three Bay 4 accelerometers.

The need for utilizing Bay 4 accelerometers to control Bay 4 vibrations is demonstrated by the open-loop modal responses in Figure 3. A torsional mode exists at 62 $\mathrm{rad} / \mathrm{sec}$ that is clearly observable by Bay 4 but does not appear in the frequency response data of Bay 3. Any feedback configuration utilizing only Bay 3 sensors fails to provide information to the controller about the torsional mode dynamics at this frequency. Consequently, the controller can not properly cancel these dynamics as is demonstrated by the poor closed-loop performance.

Output feedback controllers are generated using $D-K$ iteration for vibration attenuation of the Bay 4 accelerometers. Separate controllers are designed for 2 different feedback configurations. The first controller, $K_{3}^{4}$, will use the 3 sensors in Bay 3 to control vibration in Bay 4. The second controller, $K_{4}^{4}$, will feedback the 3 sensors in Bay 4 to attenuate vibration in Bay 4. Magnitude plots are given for $K_{3}^{4}$ in Figure $9 \mathrm{a}$ and for $K_{4}^{4}$ in Figure $9 \mathrm{~b}$.
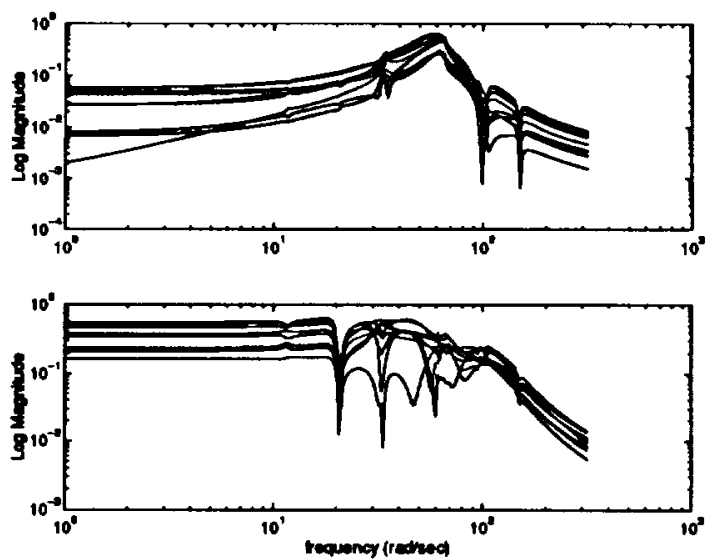

Figure 9: Magnitude Gains for $K_{3}^{4}(\mathrm{a})$ and $K_{4}^{4}(\mathrm{~b})$

Robust stability for the linear plant model with each controller with respect to the uncertainty description in Figure 1 is computed using $\mu$. All uncertainty operators are complex and linear, time-invariant. Each controller achieves robust stability with $\mu$ values of .789 for $K_{4}^{4}$ and .707 for $K_{3}^{4}$. Robust stability $\mu$ is shown in Figure 10 . 


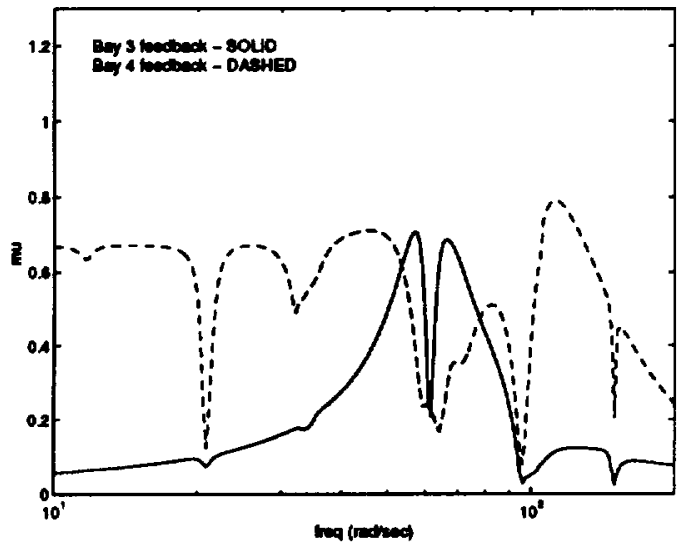

Figure 10: $\mu$ for Robust Stability

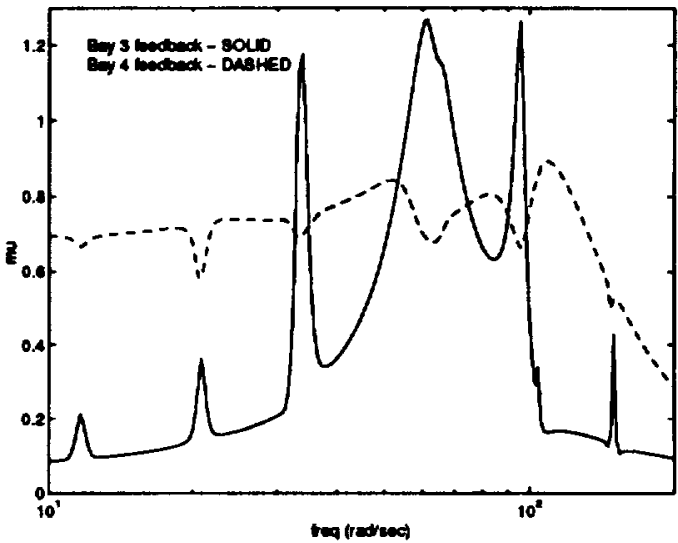

Figure 12: $\mu$ for Robust Performance
Nominal performance is also calculated for each controller as .543 for $K_{4}^{4}$ and 1.199 for $K_{3}^{4}$. The weighted norm greater than 1 indicates $K_{3}^{4}$ using the Bay 3 sensors for feedback is unable to achieve the desired performance objectives. $K_{4}^{4}$ presents a $\mu$ less than 1 and is able to achieve nominal performance. Nominal performance $\mu$ is plotted in Figure 11.

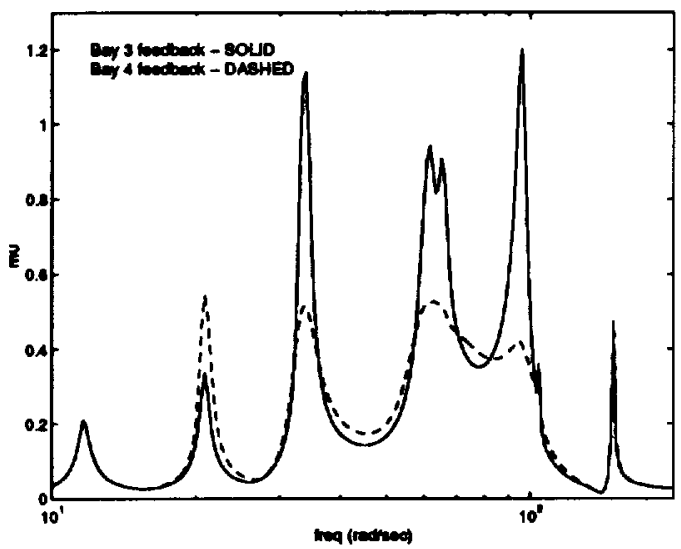

Figure 11: $\mu$ for Nominal Performance

The robust performance $\mu$ values for each controller are given in Figure 12. The $\mu$ upper bounds are computed as .982 for $K_{4}^{4}$ and 1.269 for $K_{3}^{4}$. The controller using Bay 4 feedback, $K_{4}^{4}$, is able to achieve robust performance while $K_{3}^{4}$ is unable to achieve the desired robustness goals due to its associated $\mu$ being greater than 1 .

The $\mu$ plots show controller synthesis of $K_{3}^{4}$ is driven by the unobserved torsional mode at $62 \mathrm{rad} / \mathrm{sec}$. The controller is able to lower the weighted nominal performance measure to less than 1 near this modal frequency; however, the robust stability $\mu$ is raised as a tradeoff. Robust performance $\mu$ demonstrates $K_{3}^{4}$ is unable to simultaneously achieve the desired performance and robustness goals with the peak $\mu$ occurring at $62 \mathrm{rd} / \mathrm{sec}$.
The peak $\mu$ for robust performance with $K_{4}^{4}$ occurs at $104 \mathrm{rad} / \mathrm{sec}$. This is the $4^{\text {th }}$ bending mode which is only weakly observed by Bay 4 and has a high level of additive uncertainty. $K_{4}^{4}$ does not receive sufficient information about this mode in the presence of the noise and additive uncertainty and thus the closed-loop performance is only slightly more attenuated than the open-loop performance. The controller bandwidths in Figures $9 \mathrm{a}$ and $9 \mathrm{~b}$ show $K_{3}^{4}$ is able to roll off noticeably faster than $K_{4}^{4}$ due to observance of this mode.

Implementing each controller configuration on the experimental flexible structure produces performance levels which agree with the Full Control synthesis results. Using Bay 4 accelerometers as feedback measurements allows better vibration attenuation than using Bay 3 feedbacks. Peaks gains of closed-loop transfer functions from the experimental flexible structure are presented in Figure 13. $K_{3}^{4}$ demonstrates the expected poor performance in attenuating the $62 \mathrm{rad} / \mathrm{sec}$ mode while $K_{4}^{4}$ is able to attenuate each mode to nearly equal peak gains as expected by the $\mu$ plots.

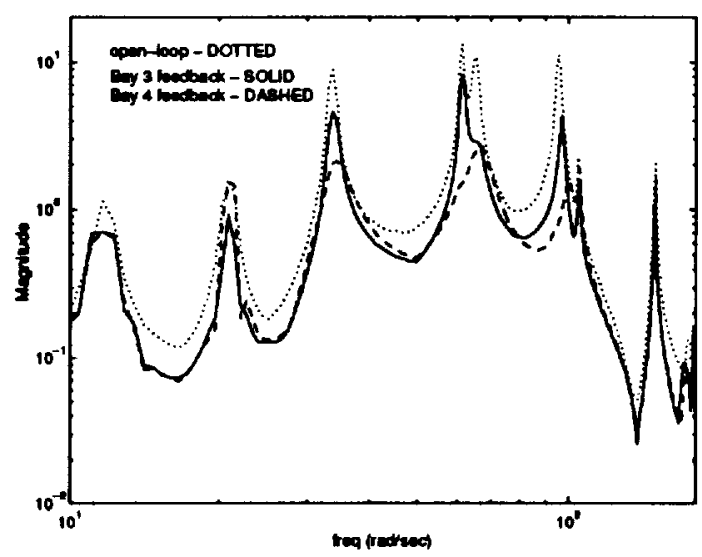

Figure 13: Experimental Closed-Loop Peak Gains for Bay 4 Accelerometers with $K_{3}^{4}(-)$ and $K_{4}^{4}(---)$ 
'G86L 7sngnV

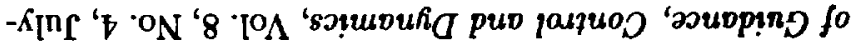

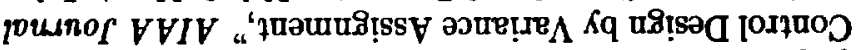

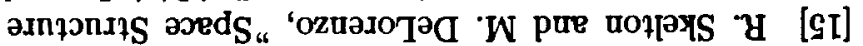

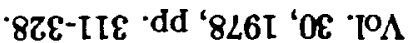

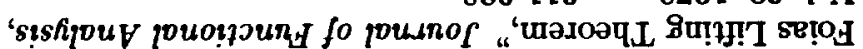

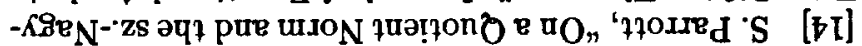
$08 \mathrm{TL}-\mathrm{LZ} \cdot \mathrm{dd}$

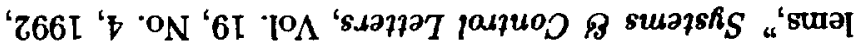

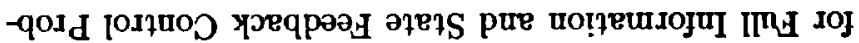

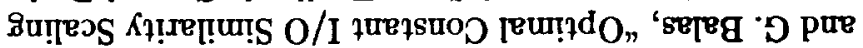

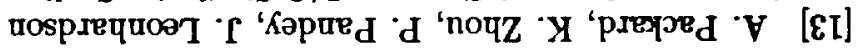

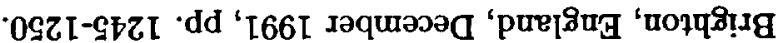

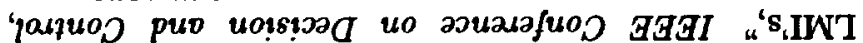

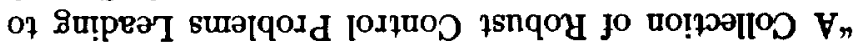

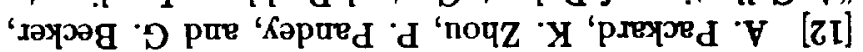

- [86\&-96

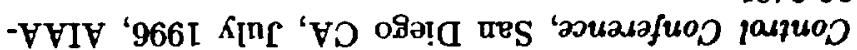

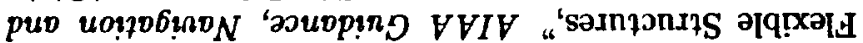

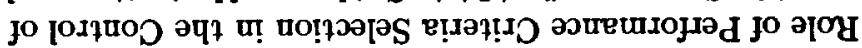
әqL" 'uosuroqL 'd pure se[eg ' $D$ 'n[sopueq[eN $\Lambda$ [tt]

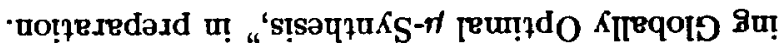
-sn notjeinsyuo jojentoy pue josues spresol qreord

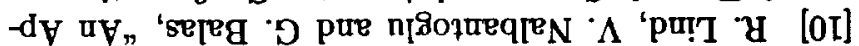

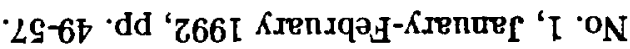

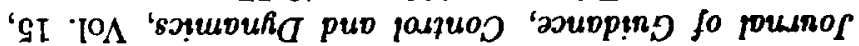

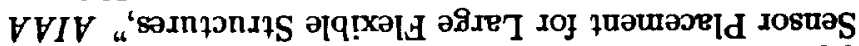

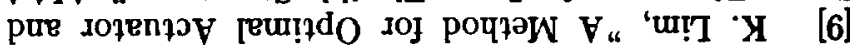

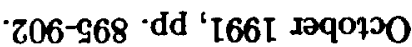

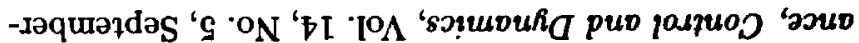

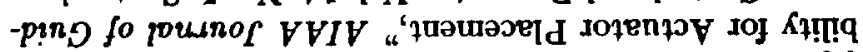

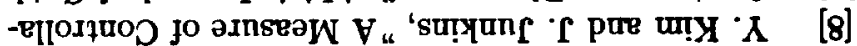

$8 \$ 7$ - $267 \cdot \mathrm{dd}$ '766I '

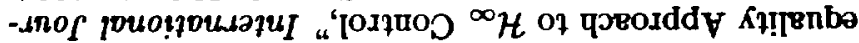

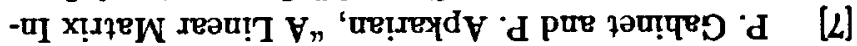

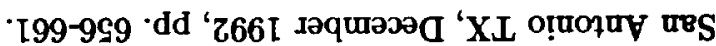

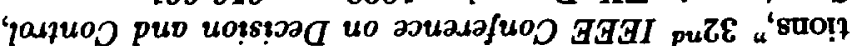
-eว!dd

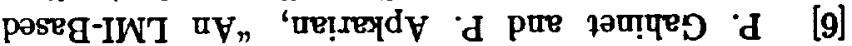

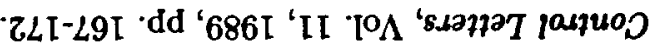

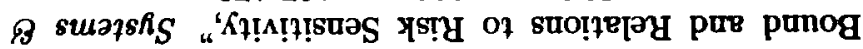

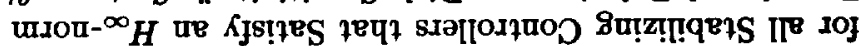

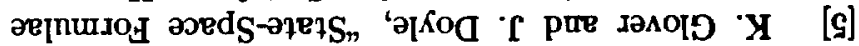

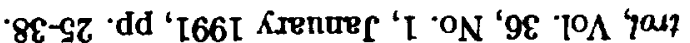
- -

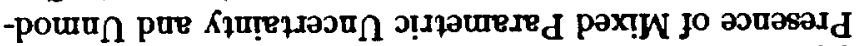

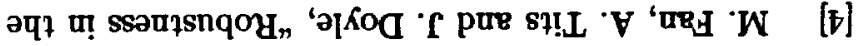

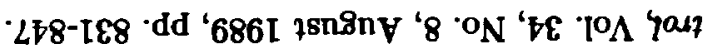
- -

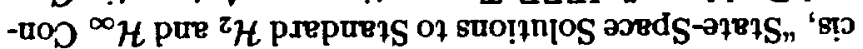

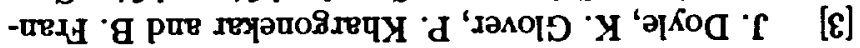

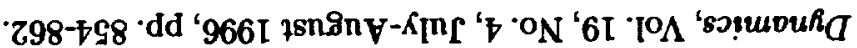

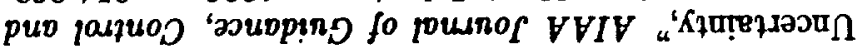

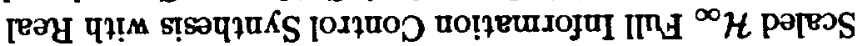

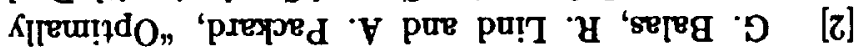

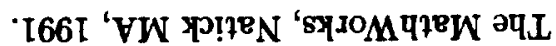

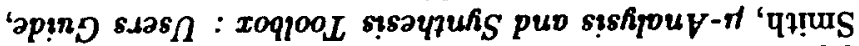

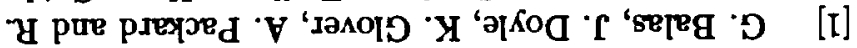
вәวนอ1әјอน

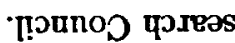

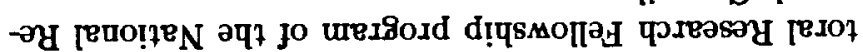

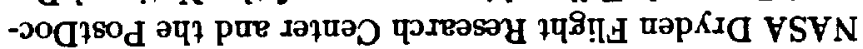

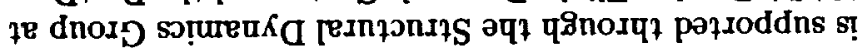
pu! I d dịsiossajod

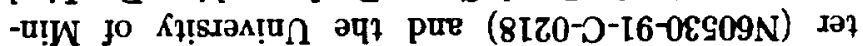

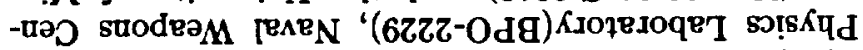

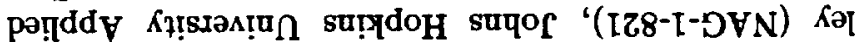

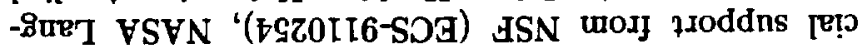

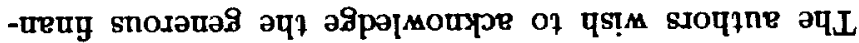

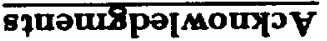

- Kq!̣!qeajasqo pure ssaupsnqoJ

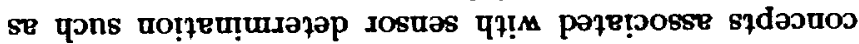

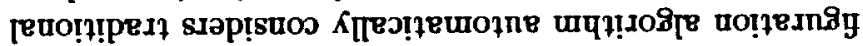

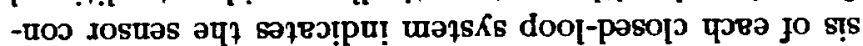

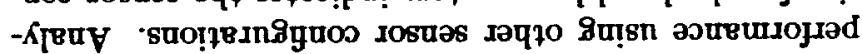

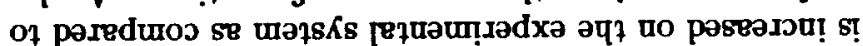

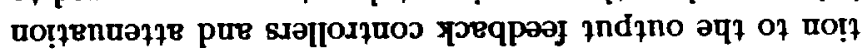

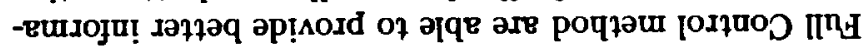

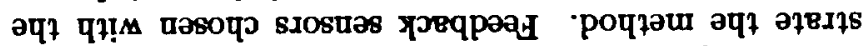

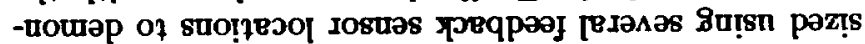

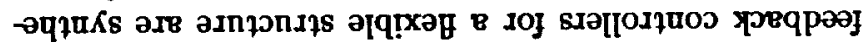
qndino 'pos pasəp!suos әप; 8uour no!̣esngyuos sosuəs

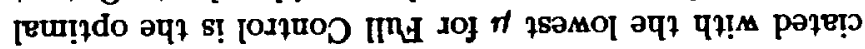

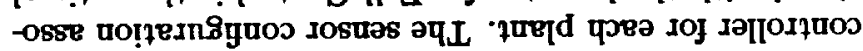

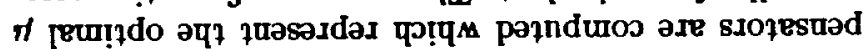

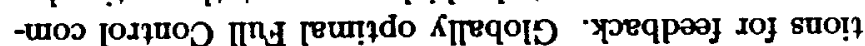

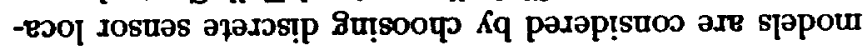

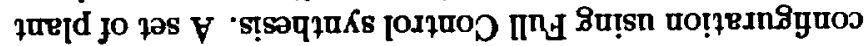

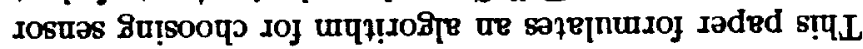

